\section{СОЗДАНИЕ ВЫСОКОВАКУУМНОЙ ЗОНЫ В АЭРОДИНАМИЧЕСКОМ СЛЕДЕ ЗА ЗАЩИТНЫМ ЭКРАНОМ В УСЛОВИЯХ ОРБИТАЛЬНОГО ПОЛЕТА НА ВЫCOTAX $\mathrm{H}=250-400 \mathrm{KM}$}

О. П. Пчеляков ${ }^{1}$, В. В. Блинов 1 , А. И. Никифоров 1 ,

Л. В. Соколов 1 , Л. Л. Зворыкин ${ }^{2}$

${ }^{1}$ Институт физики полупроводников имени А. В. Ржанова СО РАН,

г. Новосибирск, Российская Федераџия

2 ПАО «Ракетно-космическая корпораџия «Энергия» имени С. П. Королёва», г. Королев, Московская область, Российская Федерациия

На высотах орбитального полета при поперечном обтекании защитного экрана невозмущенным набегающим потоком в аэродинамическом следе за ним существует стабильная естественная область глубокого вакуума. В этой области «космического» вакуума достигаются уровни разрежения порядка $10^{-14}-10^{-10} \mathrm{Mм} \mathrm{pm} . \mathrm{cm}$. и ниже при почти полном отсутствии кислорода и углеродсодержащих компонент.

При проведении оценок достигаемых уровней разрежения предполагалосъ, что с рабочей («теневой») поверхности защитного экрана предварительно удалены сорбированные примеси, и скорости собственного газовыделения в зону следа соответствуют парциальному давлению порядка $10^{-14} \mathrm{Mм} \mathrm{pm} . \mathrm{cm}$. Этот уровень газовыделения характерен для обезгаженных металлов, применяемых в сверхвысоковакуумной технике.

Результаты оценок также показали, что из окружающей среды в зону разрежения за защитным экраном преимущественно попадают «быстрые» молекулы Не $u \mathrm{H}_{2}$, скорости теплового движения которых существенно превышают орбитальную скорость полета, и их парциальные давления на высотах $H \approx 250-400$ км на пять-шесть порядков ниже по сравнению с указанным выше парциальным давлением молекул газовыделения. Настоящая статья посвящена разработке научных основ эксперимента в условиях орбитального полета международной космической станции и обоснованию целесообразности его проведения.

Ключевые слова: космическое материаловедение, молекулярно-лучевая эпитаксия, защиттный экран, орбитальный полет, сверхвысокий вакуум.

\section{Введение}

Давление за защитным экраном (3Э) при его автономном полете в верхней атмосфере должно зависеть только от степени обезгаженности «теневой» поверхности, и уровень разрежения за $3 Э$ тогда может быть обеспечен на два-три порядка лучше предельного вакуума, достигаемого в высоковакуумных наземных технологических установках. Изменение параметров течения в аэродинамическом следе за $3 Э$ показано на рис. 1,2 . На рис. 1 приведены расчетные положения изобар в поле течения, а на рис. 2 построено изменение

(C) Пчеляков О. П., Блинов В. В., Никифоров А. И., Соколов Л. В., Зворыкин Л. Л., 2018 давления по оси течения с учетом собственного газовыделения с внутренней поверхности $3 Э$.

Как показывают расчеты, размеры и положение зоны высокого вакуума в аэродинамическом следе при $H=250-400$ км слабо зависят от высоты полета (табл. 1). На этих же высотах значения параметров $S_{H}$ и $\theta$, характеризующие условные границы аэродинамического следа за $3 Э$, зависят в основном от уровня солнечной активности и обычно изменяются в пределах $S_{H} \approx$ $4-8$, и $\theta \approx 10^{\circ}-15^{\circ}$.

При поперечных размерах $3 Э \sim 3$ м технологическая зона в области глубокого «космического» вакуума условно представляет цилиндрическую область с диаметром порядка 0,7 м и с протяженностью от днища экрана $\sim 1,5$ м, т.е. вполне допускает размещение за $3 \ni$ технологи- 
ческой, научной и контрольно-измерительной аппаратуры.

Область «космического» глубокого вакуу ма по сравнению с рабочими камерами наземных вакуумных установок обладает следующими характерными особенностями:

- продолжительность существования этой области определяется только длительностью орбитального полета;

- стабильность уровней разрежения, недостижимая в наземных условиях;

- открытость в полупространство.

\section{ВАияние собственной внешней атмосферы космических объектов}

Однако при реализации рассматриваемого проекта в составе российского сегмента (РС) международной космической станции (МКС) в условиях орбитального полета необходимо учитывать собственную внешнюю атмосферу (CBA), образующуюся около этого объекта.

При проведении на борту орбитальной станции (ОС) «Салют» и орбитального комплекса $(\mathrm{OK})$ «Мир» астрофизических, геофизических, материаловедческих и технических экспериментов установлено, что около этих объектов образуется CВА, оказывающая негативное влияние на результаты измерений и на состояние измерительных приборов. СВА представляет собой комплексное динамическое образование, включающее в себя газовую, аэрозольную, и мелкодисперсную фазы. К основным источникам формирования СВА, в частности, относятся:

- десорбция и диффузия газов и паров, адсорбированных и абсорбированных материалами внешних покрытий;

- деструкция и испарение материалов внешних покрытий;

- газовые струи работающих двигателей, выбросы газов и паров при работе системы жизнеобеспечения;

- утечка газов из гермоотсеков;

- выбросы аэрозольных и дисперсных частиц при запуске и отсечке двигателей и через дренажные системы;

- выбросы дисперсных частиц при вибрации элементов конструкции и механических ударных воздействиях.

На станции «Салют-7» в эксперименте «Астра-1» [1] при масс-спектрометрических измерениях было зафиксировано присутствие в CВА следующих газов и паров, приведенных в табл. 2.

По наблюдениям экипажей космических кораблей «Союз», ОС «Салют» и ОК «Мир» первоначально дисперсная фаза СВА образуется после отделения выводимого на орбиту объекта от последней ступени носителя, когда в поле зрения наблюдается значительное количество дисперсных частиц (по визуальным оценкам порядка нескольких тысяч с размерами 1-2 мм). При различных механических воздействиях на элементы конструкции космических объектов (вибрации, операции стыковки и расстыковки) в СВА появляется множество дисперсных частиц, размеры которых по оценкам их яркости составляют от 0,1 мм до нескольких миллиметров.

Эти дисперсные частицы фиксируются, как правило, на расстояниях до 15 м от поверхности ОК [2]. Аэрозольные и дисперсные частицы, кроме того, могут представлять собой конденсаты и продукты неполного сгорания компонентов топлива при запусках и отсечках двигателей. Для импульсных двигателей длительность переходных процессов составляет до 50 \% времени их работы, поэтому в продуктах выхлопа может находиться до половины массы используемого топлива.

В стационарном состоянии СВА, когда длительное время не производятся динамические операции (например, при гравитационной стабилизации), давление газовой фазы CBA у поверхности ОК составляет по расчётным оценкам $\sim 10^{-6}-10^{-5}$ мм рт.ст. [1], что подтверждается данными натурных измерений при использовании датчика ДВЛС. По данным этих измерений также установлено, что давление в СВА на расстоянии 3 м от поверхности ОК составляет $10^{-7}-10^{-6}$ мм рт. ст., а на расстояниях порядка 10 м от поверхности ОК не превышает значений порядка 10-7 мм рт. ст. [3].

При динамических операциях, когда работают двигательные установки, давление в СВА резко возрастает на 2-4 порядка по сравнению с фоновыми условиями, а затем релаксирует к исходному состоянию. Время релаксации возмущения давления определяется циклограммой работы двигателей, а также размерами участков поверхности элементов конструкции, попадающих в зону действия струй этих двигателей. Так, например, двигательные установки систем коррекции и ориентации ОК преимущественно работают в импульсном режиме, а направления истечения струй выбираются из условия допустимости силового и теплового воздействия на внешние поверхности элементов конструкции только периферийных зон этих струй. Через эти периферийные зоны протекает не более 2-3 \% массы газов от общего расхода, и поэтому в этом случае время релаксации СВА к фоновому состоянию, как показывают натурные измерения, составляет от нескольких до десятков минут. При работе же двигателей систем стыковки и разделения воздействию струй двигательной установки (ДУ) подвергается значительная часть внешней по- 
верхности космического объекта, и характерное время релаксации СВА к фоновому состоянию возрастает до десятков часов. В динамическом состоянии состав СВА определяется в основном продуктами выбросов из ДУ.

Эти обстоятельства указывают на необходимость применения специальных выносных устройств, обеспечивающих функционирование экспериментальной и измерительной аппаратуры за пределами СВА при проведении материаловедческих, технологических, астрофизических и геофизических исследований в составе РС МКС.

В процессе эксплуатации ОС «Салют» и ОК «Мир» проведено в общей сложности более 450 экспериментов по реализации базовых космических технологий, а также отработаны уникальные технологии по сборке и развертыванию в космосе экипажами ОС и ОК ферменных и пленочных крупногабаритных конструкций. Кроме того, отработаны методы и средства для проведения в открытом космосе регламентных и ремонтно-восстановительных работ по обслуживанию аппаратуры, по продлению ресурса ОК и его систем.

Этот имеющийся опыт позволил создать специализированную установку «Тюльпан», снабженную выносным устройством для обеспечения обтекания $3 Э$ невозмущенным набегающим потоком на высотах орбитального полета $[4 ; 5]$.

В частности, в качестве прототипа такого выносного устройства был использован задел, накопленный при создании и отработке раздвижных ферм типа «Опора».

\section{Экспериментальная установка «ТюАьпан» и перспективы ее испо ьзования}

Установка «Тюльпан» разработана в Институте электросварки им. Е. О. Патона НАН Украины и РКК «Энергия» имени С. П. Королева при участии сотрудников Института физики полупроводников имени А. В. Ржанова Сибирского отделения РАН [6-9]. Эта установка представляет собой бортовой комплекс средств обеспечения сверхвысокого вакуума для РС МКС как новой технологической среды, не имеющей наземных аналогов. В состав основных узлов экспериментальной установки «Тюльпан» входят:

- многоразовое устройство выдвижения и складывания (МУВС);

- $3 Э$ с поворотной платформой (ПП);

- платформа полезной нагрузки (ППН);

- платформа крепления;

- контрольно-измерительная и научная аппаратура (КИА и НА), устанавливаемая на ППН;

- силовые и информационные кабели.
Установка «Тюльпан» может быть использована в составе РС МКС или в составе автономного космического аппарата. Посадочное место для монтажа установки на внешней поверхности РС МКС выбирается из условия гарантированного выноса $3 Э$ с КИА и НА в невозмущенный набегающий поток при полном раскрытии МУВС. Основные цели исследований, проводимых на установке «Тюльпан» в условиях полета РС МКС, заключаются в следующем:

- измерение параметров и определение структуры течения (состав, концентрации, уровни разрежения, наличие мелкодисперсных частиц и т. д.) в ближнем аэродинамическом следе за $3 Э$ при поперечном обтекании;

- экспериментальное подтверждение возникновения в условиях орбитального полета высоковакуумной зоны в аэродинамическом следе за $3 Э$ при поперечном обтекании (с уровнями вакуума $\sim 10^{-14}-10^{-12}$ мм рт. ст. и с практически бесконечной скоростью откачки, недостижимых в наземных промышленных высоковакуумных установках);

- экспериментальное подтверждение принципиальной возможности использования этой зоны для реализации новых высоких технологий (например, выращивание полупроводниковых структур типа 3-5, 4-4 и 2-6 методом молекулярно-лучевой эпитаксии (МЛЭ), синтез которых требует уровней разрежения не менее 10-12 мм рт. ст.).

Проведение технологических исследований в области МЛЭ на установке «Тюльпан» на высотах орбитального полета позволяет использовать следующие преимущественные факторы орбитального полета:

1. Глубокий вакуум ( 10-14 $-10^{-12}$ мм рт.ст.) и практически полное отсутствие кислорода и углеродсодержащих компонент в технологической зоне за 3Э. Полученные в таких условиях эпитаксиальные слои могут обладать рекордными характеристиками по чистоте и концентрации центров безизлучательной рекомбинации. Это экспериментально доказано в 1996 г. в программе США $[10 ; 11]$.

2. Близкие к предельно возможным скорость и производительность откачки компонент рабочего молекулярного пучка, создающие уникальную возможность для сверхбыстрой смены химического состава газовой фазы в зоне роста на поверхности подложки. Указанные факторы позволяют получать гетеропереходы с идеально резкими профилями.

3. Практически полное отсутствие стенок рабочей камеры и возможность существенного уменьшения суммарной поверхности элементов технологической оснастки в зоне эпитаксиального роста. Это позволяет устранить накопление 
ранее распыленных веществ (эффект «памяти») и их неконтролируемый перенос в пленку при последующем росте других материалов и сочетать получение разнородных материалов без традиционного переноса подложки из одной ростовой камеры в другую. Кроме того, также появляется возможность увеличить количество независимых индивидуальных источников молекулярных пучков и получать сверхрезкие гетеропереходы и границы раздела между эпитаксиальными слоями, а также формировать многослойные структуры, содержащие большое число разных по составу слоев.

4. Возможность значительного увеличения расстояния от подложки до источника молекулярного пучка. Этот фактор является основным параметром, определяющим однородность слоев по площади подложек, и играет особую роль при увеличении их диаметра.

5. Возможность использования токсичных летучих жидкостей и газов (гидриды, металлорганические соединения) в качестве исходных материалов для синтеза пленок без загрязнения окружающей среды. Эти соединения быстро рассеиваются до безопасных концентраций и легко разлагаются на безопасные компоненты под действием солнечного ионизирующего излучения.

Таблица 1

Положение изобары $p \approx 10^{-12}$ мм рт. ст. на оси аэродинамического следа

\begin{tabular}{|c|c|c|c|c|}
\hline$H[$ км] & 250 & 300 & 350 & 400 \\
\hline$\tilde{x}_{\text {гр }}=x / R_{3 \ni}$ & 1,058 & 1,078 & 1,115 & 1,135 \\
\hline
\end{tabular}

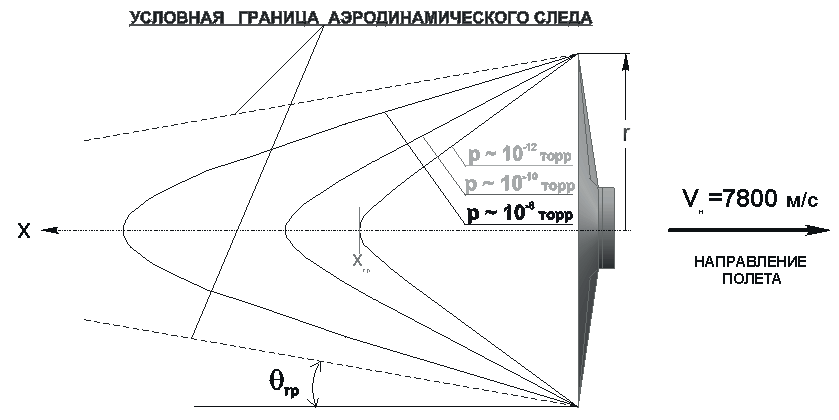

Рис. 1. Уровни разрежения за $3 Э$ при поперечном обтекании в условиях орбитального полета

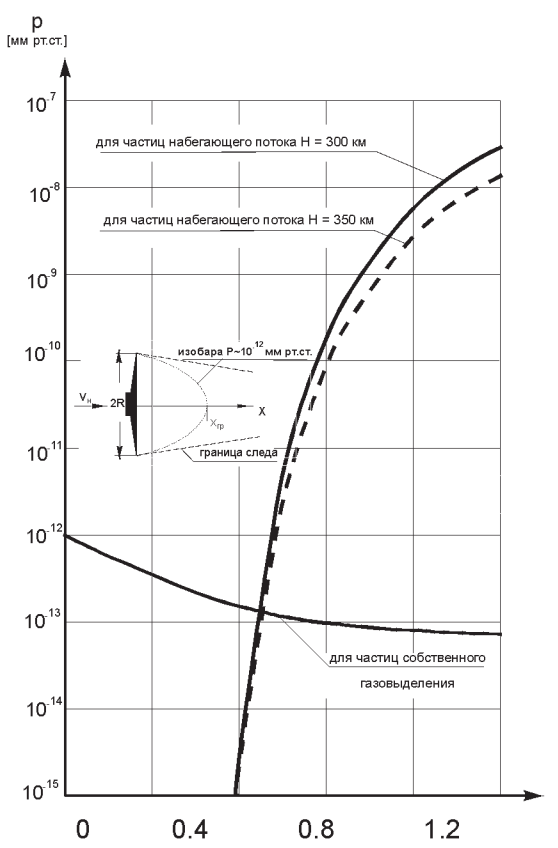

Рис. 2. Распределение давления по оси аэродинамического следа за $3 Э$ при поперечном обтекании в условиях орбитального полета

Таблица 2

Состав газовой фазы CBA

\begin{tabular}{|c|c|c|c|c|c|}
\hline M & Нейтралы & Ионы & $\mathrm{M}$ & Нейтралы & Ионы \\
\hline 2 & $\mathrm{H}_{2}$ (ДУ) & & 28 & 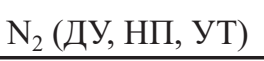 & $\mathrm{N}_{2}+($ ДУ, НП, УТ) \\
\hline 4 & Не (ДУ) & & 28 & СО (ДУ) & \\
\hline 14 & N (ДУ, НП) & N+ (ДУ, НП) & 30 & NO (ДУ) & NO+ (ДУ) \\
\hline 16 & $\mathrm{CH}_{4}($ ДУ) & & 32 & $\mathrm{O}_{2}(\mathrm{H \Pi})$ & $\mathrm{O}_{2}+(\mathrm{H \Pi})$ \\
\hline 17 & $\mathrm{O}(\mathrm{HП)}$ & $\mathrm{O}+(\mathrm{H \Pi})$ & 40 & $\operatorname{Ar}(\mathrm{H \Pi )}$ & \\
\hline 18 & $\mathrm{H}_{2} \mathrm{O}$ & $\mathrm{H}_{2} \mathrm{O}+$ & 44 & $\mathrm{CO}_{2}$ & $\mathrm{CO}_{2}+$ \\
\hline 19 & & $\mathrm{H}_{3} \mathrm{O}+(Д У)$ & 103 & Фреоны (21) & \\
\hline
\end{tabular}


Здесь М - массовое число составляющей газовой фазы; Г, ДУ, НП и УТ - соответствуют наименованиям источников СВА (газовыделение, двигательные установки, набегающий поток и утечки из отсеков и систем ОК). В табл. 2 отсутствуют данные по «тяжелым» компонентам из-за ограниченной разрешающей способности по массовым числам, использованной в эксперименте «Астра-1» масс-спектрометрической аппаратуры.

\section{Закмючение}

Результаты исследований сибирских ученых показали, что развивать крупномасштабное производство наноструктурных высокоэффективных космических солнечных батарей в России чрезвычайно важно для обеспечения программ космических исследований и обороноспособности страны, для развития систем космической связи, информационных и информационно-управ- ляющих систем. В настоящее время в Институте физики полупроводников имени А. В. Ржанова СО РАН в сотрудничестве с красноярскими учеными из Красноярского научного центра СО РАН и предприятия АО «Информационные спутниковые системы» имени академика М. Ф. Решетнёва» планируется создание оборудования МЛЭ для выращивания структур высокоэффективных преобразователей солнечного света в электричество. По заказу Роскосмоса разработан и прошел приемку эскизный проект установки для синтеза в условиях космического пространства нового материала для высокоэффективных солнечных батарей.

Перед нами стоит задача разработать отечественную импортонезависимую технологию и оборудование для создания солнечных элементов космических аппаратов и организовать их производство в Российской Федерации, для чего целесообразно дальнейшее развитие сверхвысоковакуумной технологии МЛЭ.

\section{Список литературы}

1. Зворыкин Л. Л., Котов В. М., Крылов А. Н. Моделирование взаимодействия потока сильно разреженного газа с обтекаемой поверхностью / Труды Х Всесоюзной конференции по динамике разреженных газов. М. Изд-во МЭИ. 1991. С. 31-39.

2. Pchelyakov O. P., Dvurechensky A. V., Latyshev A. V., Aseev A. L. Ge/Si heterostructures with coherent Ge quantum dots in silicon for applications in nanoelectronics // Semiconductor Science and Technology, 2011, vol. 26, no. 1, pp. 14-27. doi: 10.1088/0268-1242/26/1/014027

3. Климук П. И.,Забелина И. А., Гоголев В. П. Визуальные наблюдения и загрязнения оптики в космосе. Л. Машиностроение. 1983.

4. Зворыкин Л. Л., Мишина Л. В., Пярнпуу А. А. Моделирование взаимодействия разреженного газа с твердой поверхностью / Препринт ВЦ АН СССР. М. 1988.

5. Mishina L. V., Krylov A. N., Pyarnpuu A. A., Zvorykin L. L. Kinetic Modeling of Flows near Complex Form Bodies. Rarefied Gas Dynamics. New York, 1991, pp. 1391-1397.

6. Пчеляков О. П., Ольшанецкий Б. З., Гутаковский А. П. Эпитаксия гетероструктур на кремнии в условиях космоса (предложение на проведение исследований по программе РАН на ОК «Мир»). ИФП СО РАН, 1996.

7. Нусинов М. Д. Воздействие и моделирование космического вакуума. М. 1982.

8. Беляков И. Т., Борисов Ю. Д. Технология в космосе. М. 1974.

9. Валиев К., Орликовский А. Технологии СБИС. Основные тенденции развития // Электроника. Наука. Технология. Бизнес. 1996. № 5-6, С. 3-10.

10. Ignatiev A. The wake shield facility and space-based thin film science and technology // Earth Space Revew, 1995, vol. 2, no. 2, pp. 10-17.

11. Ignatiev A., Freundlich A., Pchelyakov O., Nikiforov A., Sokolov L., Pridachin D., Blinov V. Molecular Beam Epitaxy in the Ultravacuum of Space: Present and Near Future // From Research to Mass Production, 2018, pp. 741-749. doi: 10.1016/B978-0-12-812136-8.00035-9 


\title{
THE CREATION OF A HIGH VACUUM ZONE IN THE AERODYNAMIC WAKE BEHIND A PROTECTIVE SCREEN IN THE CONDITIONS OF ORBITAL FLIGHT ON THE ALTITUDE $\mathrm{H}=250-400 \mathrm{KM}$
}

\author{
O. P. Pchelyakoví, V. V. Blinov', A. I. Nikiforov'1, \\ L. V. Sokolov', L. L. Zvorykin ${ }^{2}$
}

IRzhanov Institute of Semiconductor Physics SB RAS, Novosibirsk, Russian Federation ${ }^{2}$ S. P. Korolev Rocket and Space Public Corporation Energia, Korolev, Russian Federation

At the heights of orbital flight at the transverse flow around the protective shield unperturbed oncoming flow in the aerodynamic wake behind it there is a stable natural region of high vacuum. In this area of the «space» vacuum the levels of vacuum of the order are reached $10^{-14}-10^{-10} \mathrm{~mm}$ Mercury and below in the almost complete absence of oxygen and carbonaceous components.

When assessing the achieved levels of dilution, it was assumed that the working («shadow») protective shield surface pre-adsorbed impurities are removed, and the speed of its own gas in the area of the track does not exceed this level, which corresponds to a partial pressure of about $10^{-14} \mathrm{~mm}$ Mercury.

The results of the evaluations also showed that from the environment into the vacuum zone for protective shield mostly fall into the "fast» molecules of $\mathrm{He}$ and $\mathrm{H}_{2}$, the thermal velocity which significantly exceed the orbital velocity of flight, and their partial pressure at altitudes of $250-400 \mathrm{~km}$ five to six orders of magnitude lower compared to the above-mentioned partial pressure of molecules of gas. This article is devoted to the development of the scientific basis of the experiment in the conditions of the orbital flight of the international space station and the justification of its feasibility.

Keywords: space materials science, molecular beam epitaxy, shield, orbital flight, ultrahigh vacuum.

\section{References}

1. Zvorykin L. L., Kotov V. M., Krylov A. N. Modelirovanie vzaimodejstviya potoka sil'no razrezhennogo gaza $s$ obtekaemoj poverhnost'yu [Simulation of the interaction of a stream of highly rarefied gas with a streamlined surface]. Moscow, MEI, 1991, pp. 31-39. (In Russian)

2. Pchelyakov O. P., Dvurechensky A. V., Latyshev A. V., Aseev A. L. Ge/Si heterostructures with coherent Ge quantum dots in silicon for applications in nanoelectronics // Semiconductor Science and Technology, 2011, vol. 26, no. 1, pp. 14-27. doi: 10.1088/0268-1242/26/1/014027

3. Klimuk P. I, Zabelina I. A., Gogolev V. P. Vizual'nye nablyudeniya i zagryazneniya optiki v kosmose [Visual observations and contamination of optics in space]. Mechanical Engineering, 1983. (In Russian)

4. Zvorykin L. L., Mishina L. V., Pyarnpuu A. A. Modelirovanie vzaimodejstviya razrezhennogo gaza s tverdoj poverhnost'yu [Modeling the interaction of a rarefied gas with a solid surface]. Moscow, 1988. (In Russian)

5. Mishina L. V., Krylov A. N., Pyarnpuu A. A., Zvorykin L. L. Kinetic Modeling of Flows near Complex Form Bodies. Rarefied Gas Dynamics. New York, 1991, pp. 1391-1397.

6. Pchelyakov O. P., Olshanetsky B. Z., Gutakovsky A. P. Epitaksiya geterostruktur na kremnii v usloviyah kosmosa (predlozhenie na provedenie issledovanij po programme RAN na OK «Mir») [Epitaxy of heterostructures on silicon in space conditions (proposal for research under the program of the Russian Academy of Sciences on the OK Mir)]. IPhS SB RAS, 1996. (In Russian)

7. Nusinov M. D. Vozdejstvie i modelirovanie kosmicheskogo vakuuma [The Impact and Modeling of the Cosmic Vacuum]. Moscow, 1982. (In Russian)

8. Beliakov I. T., Borisov Yu. D. Tekhnologiya v kosmose [Technology in space]. Moscow, 1974. (In Russian)

9. Valiev K., Orlikovsky A. Tekhnologii SBIS. Osnovnye tendencii razvitiya [VLSI Technologies. Major development trends] // Electronics. Science. Technology. Business, 1996, no. 5-6, pp. 3-10. (In Russian)

10. Ignatiev A. The wake shield facility and space-based thin film science and technology // Earth Space Revew, 1995, vol. 2, no. 2, pp. 10-17.

11. Ignatiev A., Freundlich A., Pchelyakov O., Nikiforov A., Sokolov L., Pridachin D., Blinov V. Molecular Beam Epitaxy in the Ultravacuum of Space: Present and Near Future // From Research to Mass Production, 2018, pp. 741-749. doi: 10.1016/B978-0-12-812136-8.00035-9 